\title{
Praxis, Theorie und Wissenschaft im Selbstverständnis des städtischen Arztes Christoph Jacob Trew (1695-1769)
}

Thomas Schnalke

\section{Summary}

In addition to universities and courts, large towns were regarded as attractive locations for a successful career by physicians in the 18th century. This study reveals the spectrum of practical and scientific activities of a "physicus" like Christoph Jacob Trew (1695-1769) who lived in the German town of Nuremberg. Emphasis is put on his attitude towards the meaning of medical practice, theory, and science in his work. Letters and drafts which form the basis of this study prove their usefulness as sources for medicohistorical studies on the professional world of practicing physicians in the 18 th century.

\section{Zusammenfassung}

Neben der Universität und dem Hof erachtete der Arzt des 18. Jahrhunderts für sein berufliches Wirken vor allem eine grössere Stadt als einen erstrebenswerten Ort, wo er sich bei einigem Geschick eine sichere Existenz aufbauen und gegenüber seinen Patienten Achtung und Anerkennung erwerben konnte. Darüber hinaus war es ihm möglich, im bürgerlich-urbanen Milieu als medizinischer Lehrer, Forscher und Publizist ein quasi akademisches Leben zu führen. Dieser Beitrag stellt die Entfaltungsmöglichkeiten eines städtischen Arztes am Beispiel des Nürnberger Physikus Christoph Jacob Trew (1695-1769) dar und untersucht dessen Auffassung über die Bedeutung von medizinischer Praxis, Theorie und Wissenschaft für sein Schaffen. Die

Priv.-Doz. Dr. med. Thomas Schnalke, Institut für Geschichte der Medizin, Friedrich-Alexander-Universität Erlangen-Nürnberg, Glückstr. 10, D-91054 Erlangen 
Arbeit basiert vor allem auf Briefen und Briefentwürfen, die sich als Quellen für medizinhistorische Untersuchungen der beruflichen Welt des praktischen Arztes im 18. Jahrhundert anbieten.

«[...] alleine ein jeder wird auch gestehen müßen daß wann einmal ein systematischer grund in theorica geleget ist dieselbigen [Schwierigkeiten in der Praxis] durch die praxin selbsten am besten gehoben werden, ja daß die praxis vielfälltig die theorie verbeßert und erläutert». ${ }^{1}$ Mit diesen Worten versuchte der angesehene Nürnberger Arzt Christoph Jacob Trew im Jahre 1732 den jungen Doktor der Medizin, Georg Leonhard Huth, dazu zu bewegen, seine Studienreise zu beenden und in seine Heimatstadt an der Pegnitz zurückzukehren, um dort eine ärztliche Praxis zu eröffnen. Trew übermittelte seine Gedanken in einem Brief, zu welchem sich im Briefarchiv der Erlanger Universitätsbibliothek ein Entwurf erhalten hat, der Einblicke in das berufliche Selbstverständnis eines in einer grösseren Stadt tätigen praktischen Arztes des 18. Jahrhunderts gewährt.

Die städtische Medizin im Jahrhundert der Aufklärung wird von der aktuellen fachhistorischen Forschung weitgehend ausgeblendet. Neuere ideengeschichtliche Ansätze gelangen zwar zu der Feststellung, dass die praktische Dimension der Medizin in der ersten Hälfte des 18. Jahrhunderts allgemein an Bedeutung gewann ${ }^{2}$, ihre Perspektive bleibt jedoch, nicht zuletzt bedingt durch die Bevorzugung gedruckter Quellen, auf die Entwicklung theoretischer Gedankengebäude in den Grenzen der universitären und akademischen Sphäre der Medizin gerichtet. Demgegenüber wenden sich jüngere sozial-, alltags- und mentalitätsgeschichtliche Studien in der Auswertung eines von ihren Autoren geforderten heterogenen Quellenbestandes zwar ausdrücklich auch der praktischen Welt der Medizin in diesem Zeitraum zu, jedoch untersuchen sie mit Vorliebe den Status der Abnehmer und der nicht akademisch gebildeten Anbieter von Medizin. ${ }^{3}$

1 Trew Chr. J. 378, 17. 11. 1732. - Der Nachweis der hier wiedergegebenen Zitate aus Briefen und Briefentwürfen folgt der Verzeichnung in Schmidt-Herrling, Eleonore: Die Briefsammlung des Nürnberger Arztes Christoph Jacob Trew (1695-1769) in der Universitätsbibliothek Erlangen. Erlangen, Universitätsbibliothek, 1940.

2 Vgl. Geyer-Kordesch, Johanna: Medizinische Fallbeschreibungen und ihre Bedeutung in der Wissensreform des 17. und 18. Jahrhunderts. In: Medizin, Gesellschaft und Geschichte. Jahrbuch des Instituts für Geschichte der Medizin der Robert Bosch Stiftung 9 (1990) 7-19; Müller, Ingo Wilhelm: Iatromechanische Theorie und ärztliche Praxis im Vergleich zur galenistischen Medizin. Stuttgart, Steiner, 1991.

3 Vgl. für den deutschen Sprachraum Jütte, Robert: Ärzte, Heiler und Patienten. Medizinischer Alltag in der frühen Neuzeit. München, Artemis \& Winkler, 1991; Sander, Sabine: Handwerkschirurgen. Sozialgeschichte einer verdrängten Berufsgruppe. Göttingen, Vandenhoeck \& Ruprecht, 1989; Loetz, Francisca: Vom Kranken zum Patienten. «Medikalisierung» und medizinische Vergesellschaftungen am Beispiel Badens 1750-1850. Stuttgart, Steiner, 1993. 
Wie konstituiert sich hingegen die Lebenswelt der praktischen Ärzte, die im 18. Jahrhundert im europäischen Raum neben einer Anstellung an verschiedenen Höfen vor allem in den Städten in grösserer Dichte ihr Auskommen suchten? ${ }^{4}$ Was kennzeichnet ihr medizinisches Denken, Handeln und Streben? Um auf diese Fragen Antworten mit allgemeinerem Aussagewert zu finden, ist es notwendig, möglichst authentische und somit vor allem archivalische Quellen zu erschliessen. Als eine interessante Quellengattung bieten sich in diesem Zusammenhang Briefe und Briefwechsel an, die sich von ärztlichen Autoren dieser Zeit in teilweise grossen Corpora erhalten haben. ${ }^{5}$ Im Mittelpunkt der vorliegenden Arbeit stehen daher zwei längere Passagen aus Briefentwürfen Christoph Jacob Trews ${ }^{6}$, die exemplarisch das Selbstbild eines ärztlichen Individuums erhellen.

Georg Leonhard Huth entstammte einer in Nürnberg ansässigen Kaufmannsfamilie. ${ }^{7}$ Nach Abschluss seines Medizinstudiums an der Universität Altdorf begab er sich 1728 auf eine Studienreise, die ihn über Strassburg nach Paris und Leiden führte. Während der Abwesenheit von seiner Heimatstadt stand er in brieflichem Kontakt mit Christoph Jacob Trew, der zu dieser Zeit als Arzt in Nürnberg fest etabliert und angesehen war. ${ }^{8}$ Huth hatte schon zu seinen Studienzeiten in Trew seinen «werthesten Patron $»^{9}$ gefunden, dem nun auch der weitere berufliche Werdegang des um zehn Jahre jüngeren Kollegen am Herzen lag. So empfahl Trew seinem Schützling, den Aufenthalt in Strassburg kürzer zu halten, um die Weiterbildungsangebote in Frankreich

4 Vgl. Stürzbecher, Manfred: Über die medizinische Versorgung der Berliner Bevölkerung im 18. Jahrhundert. In: ders. (Hrsg.): Beiträge zur Berliner Medizingeschichte. Quellen und Studien zur Geschichte des Gesundheitswesens vom 17. bis zum 19. Jahrhundert. Berlin, de Gruyter, 1966, 67-155; Russel, Andrew W. (Hrsg.): The Town and State Physician in Europe from the Middle Ages to the Enlightenment. Wolfenbüttel, Hubert, 1981; Jütte (1991, s. Anm. 3) 17-32; Porter, Dorothy und Roy Porter: Patient's Progress. Doctors and doctoring in eighteenth-century England. Cambridge, Polity, 1989.

5 Vgl. Schmidt-Herrling (s. Anm. 1); Boschung, Urs: Albrecht von Hallers Korrespondenz und ihre Erschliessung. Gesnerus 46 (1989) 211-227; Döhring, Detlef: Der Nachlass von Christian Gottlieb Ludwig (1709-1773) in der Universitätsbibliothek Leipzig. Medizinhistorisches Journal 27 (1992) 113-125.

6 Die Wiedergabe der Textpassagen aus den hier zitierten Briefen und Briefentwürfen folgen weitgehend den «Empfehlungen zur Edition frühneuzeitlicher Texte», in: Berichte zur Wissenschaftsgeschiche 4 (1981) 167-181.

7 Zur Biographie Georg Leonhard Huths (1705-1761) vgl. Will, Georg Andreas: Nürnbergisches Gelehrten-Lexicon. Nürnberg, Altdorf, Schüpfel, 1756, Bd. 2, S. 210-213; ders.: Nürnbergisches Gelehrten-Lexicon [...] fortgesetzet von Christian Conrad Nopitsch. Altdorf, Eigenverlag, 1802, Bd. 5, S. 145-146.

8 Zur Biographie Christoph Jacob Trews vgl. Schmidt-Herrling, Eleonore: Christoph Jacob Trew, 1695-1769. Medizinische Monatsschrift 7 (1953) 382-385; Pirson, Julius: Der Nürnberger Arzt und Naturforscher Christoph Jakob Trew (1695-1769). Mitteilungen des Vereins für Geschichte der Stadt Nürnberg 44 (1953) 448-575.

9 Huth, G. L. 2, 25. 4. 1729. 
und Holland intensiver nutzen zu können. ${ }^{10}$ Als sich Huth jedoch entschloss, für längere Zeit in Strassburg zu verweilen, wo er ideale Lernbedingungen auf den Gebieten der Anatomie und Geburtshilfe vorgefunden zu haben glaubte $^{11}$, teilte sein Mentor diese Auffassung und vertrat sie auch gegenüber Huths Brüdern, die auf eine baldige Rückkehr des jungen Arztes drängten. ${ }^{12}$ Unterdessen setzte sich Trew dafür ein, dass dem Reisenden ein Platz im Nürnberger Collegium medicum reserviert wurde. ${ }^{13}$ Als Huth seine Studienreise jedoch immer weiter ausdehnte und die Korrespondenz mit Trew mehr als eineinhalb Jahre aussetzte, griff sein Nürnberger Patron schliesslich zur Feder, um ihm eine rasche Beendigung seines Auslandsaufenthalts nahezulegen. Trew kleidete sein Anliegen in folgende Worte: ${ }^{14}$

\begin{abstract}
"Als vormals diese [anatomische und chirurgische] Profession [in Altdorf] vacant ware, hielte ich es vor ein unglück daß sie mir nicht zu theil worden, Gott aber hat mich nachmals in einen solchen Stande gesetzt daß ich solchen damit nicht vertauschen wollte. Man hat auch hier in Nürnberg Gelegenheit mit seinen erlangten wißenschafften dem nechsten auf allerley weiße nützen zu können. Die Praxis, als das haupt werck so damit verknüpfet ist, ist zwar mühsam, doch fordert sie nicht mehr als alles andere das man sich will angelegen seyn lassen, und erleichtert sich durch sich selbsten. Wie mich Dero Herrn Brüdere berichtet haben, so stellen sich Euer HochEdel dieselbe allzu schwehr vor. Nun muß ich zwar wie ein jeder aufrichtige und gewißenhaffter medicus, aus eigener erfahrung bekennen daß sich schwührigkeiten genug dabey finden, alleine ein jeder wird auch gestehen müßen daß wann einmal ein systematischer grund in theorica geleget ist dieselbigen durch die praxin selbsten am besten gehoben werden, ja daß die praxis vielfälltig die theorie verbeßert und erläutert. Der anfang bleibet hier wie sonsten allezeit schwehr, wann man ihn auch noch so lang verschiebet. Im übrigen ist das noch das beste daß nicht alle Kranckheiten consilium ex arena erfordern, hat man sich die methode, diejenige, wo periculum in mare ist, zu tractiren familiari gemacht, so ist bey denen andern zeit genug übrig durch nachdencken und nachschlagen der autorum die schwührigkeiten zu heben, und man kan in casu dubio mit gutem gewißen bey der ersten visite sich mit generalioribus behelfen.»
\end{abstract}

Huth sah sich durch diese Zeilen Trews nicht dazu veranlasst, seine Studienreise sofort abzubrechen. Erst ein knappes Jahr später kehrte er nach Nürnberg zurück, um dort eine Praxis zu eröffnen..$^{15}$

Christoph Jacob Trew leitet seine grundsätzlichen Ausführungen mit einem Hinweis auf seine eigene Biographie ein. ${ }^{16} \mathrm{Im}$ Jahr 1720 war er in aller Eile von seiner dreijährigen Studienreise zurückgekehrt, da ihm in Altdorf der Lehrstuhl für Chirurgie und Anatomie in Aussicht gestellt worden war.

10 Trew sprach diese Empfehlung gegenüber Huths älterem Bruder Philipp Ludwig (1696-1752) aus, der seit 1724 als angesehener Jurist in Nürnberg lebte; zur Person Philipp Ludwig Huths vgl. Will (s. Anm. 7) 208-210; Will und Nopitsch (s. Anm. 7) 145.

11 Vgl. Huth G. L. 2, 25. 4. 1729.

12 Vgl. Trew Chr. J. 376, 28. 7.1729.

13 Vgl. Trew Chr. J. 377, vor dem 14. 2. 1731. Huth bedankte sich für diese Gefälligkeit in seinem Gegenbrief; vgl. Huth G. L. 14.2.1731.

14 Trew Chr. J. 378, 17.11.1732.

$15 \mathrm{Vgl} \mathrm{Huth} \mathrm{G.} \mathrm{L.} \mathrm{6,} \mathrm{1.} \mathrm{5.} \mathrm{1733;} \mathrm{Huth} \mathrm{G.} \mathrm{L.} \mathrm{7,} \mathrm{27.} \mathrm{7.} 1733$.

$16 \mathrm{Vgl}$. Anm. 8. 
Zu seiner grossen Enttäuschung zog ihm der Nürnberger Magistrat jedoch den Hallenser Dozenten Johann Heinrich Schulze vor. ${ }^{17}$ Er selbst fand noch im gleichen Jahr Aufnahme im reichsstädtischen Collegium medicum. 1721 liess er sich in Nürnberg nieder und festigte in den folgenden Jahren seine berufliche Stellung so weit, dass er Angebote, an einen Hof oder eine Universität zu wechseln, wiederholt ausschlug. In seinem Brief an Huth brachte er sein Bekenntnis für die Stadt als ideale ärztliche Arbeitsstätte auf den Punkt, als er formulierte: «Man hat auch hier in Nürnberg Gelegenheit mit seinen erlangten wißenschafften dem nechsten auf allerley weiße nützen zu können.» ${ }^{18}$

Die Ausübung der ärztlichen Praxis liefert für Trew die beste Gelegenheit, die sich einem Arzt eröffnet, um seine erlangten Wissenschaften nutzbringend anzuwenden. Dem Nürnberger Arzt gilt die Praxis als das «haupt werck», als das Fundament seines medizinischen Wirkens. Zwar sei jede Praxis mühsam, jedoch müsse sich der Arzt für diese öffnen und sich auf sie einlassen, denn erst durch sie werde der Prozess des Erfahrungsammelns in Gang gesetzt, in welchem sich die Praxis durch sich selbst erleichtere. Praktisches Erfahrungswissen komme jedoch nicht nur der Praxis zugute, sondern wirke auch auf die medizinische Theorie zurück. Das theoretische Wissen werde durch sie verbessert und erläutert.- So gesehen stellt Trew die aus der Praxis gewonnene Erfahrung und damit die ärztliche Praxis selbst in ihrer Bedeutung ein Stück weit über die medizinische Theorie.

Die Theorie lässt sich mithilfe der Praxis allerdings erst dann vorantreiben und erklären, wenn der Arzt den Schritt in die Praxis tatsächlich wagt. Trew beschreibt den beruflichen Anfang als einen kritischen Moment im Werdegang des ärztlichen Praktikers. Theoretisch ausgebildet, steht der junge Arzt ohne Erfahrung vor den Anforderungen der Praxis. Um in den klinischen Alltag zu finden, empfiehlt Trew, in der Begegnung mit dem Patienten einige Verhaltens- und Handlungsstrategien zu befolgen, die vorrangig darauf abzielen, Notfallsituationen erkennen und beherrschen zu lernen und in allen übrigen Fällen die Zeit für sich arbeiten zu lassen, um durch ruhiges Nachdenken und -lesen zum bestmöglichen ärztlichen Vorgehen zu gelangen.

Bei aller Wertschätzung der medizinischen Praxis geht Trew in der Entwicklung seiner Ansichten von einer schwierigen Klinik, einem mit allerlei Mühsal befrachteten ärztlichen Alltag aus, ohne jedoch die Widrigkeiten ge-

17 Zu Leben und Werk Johann Heinrich Schulzes (1687-1744) vgl. zuletzt Kaiser, Wolfram und Arina Völker (Hrsg.): Johann Heinrich Schulze (1687-1744) und seine Zeit. Halle (Saale), 1987.

18 Trew Chr. J. 378, 17. 11. 1732. 
nauer zu benennen, mit welchen sich ein ärztlicher Praktiker im 18. Jahrhundert, zumal in den ersten Jahren seiner beruflichen Tätigkeit, konfrontiert sah. Auch seine Empfehlungen für den Berufsanfänger reflektieren die Hindernisse und Widerstände in der Praxis nur indirekt, so dass die Frage bleibt, auf was sich ein Arzt wie etwa Georg Leonhard Huth tatsächlich einlassen musste, wenn er sich in einer Stadt niederliess.

Wollte ein Arzt im genannten Zeitraum in Nürnberg eine Praxis eröffnen, so bedurfte es zunächst der Approbation durch den lokalen Magistrat und der Aufnahme in das örtliche Collegium medicum. ${ }^{19}$ Hierfür galten der Studienabschluss, die Promotion, eine längere Studienreise sowie das Bürgerrecht als unabdingbare Voraussetzungen.

In Nürnberg konkurrierte ein ärztlicher Berufsanfänger mit einer grösseren Anzahl von bereits eingeführten Kollegen um eine insgesamt nicht sehr grosse Gruppe zahlungskräftiger Patienten. Die organisierte Ärzteschaft Nürnbergs setzte sich seit der Einführung der ersten lokalen Medizinalordnung im Jahr 1592 aus vergleichsweise vielen medizinischen Doktoren zusammen. Zwischen 1670 und 1710 schnellte die Mitgliederzahl von 10 auf 26 hoch, während die Bevölkerung in diesem Zeitraum, bedingt durch den andauernden wirtschaftlichen Niedergang der Stadt, kontinuierlich abnahm und bis zum Ende des 18. Jahrhunderts nur noch die Hälfte der einst 50000 Einwohner betrug. ${ }^{20}$ Das Zielpublikum akademisch gebildeter Ärzte konzentrierte sich dabei vorrangig auf die Mitglieder des staatstragenden Patriziats und der reicheren Kaufmannsschicht.

Die Ausrichtung auf eine sozial höher gestellte Patientenschaft sowie die Konkurrenzsituation unter den Ärzten und Heilern zwang den Praktiker in Abhängigkeiten, zum einen in ökonomische, zum anderen aber auch in therapeutische. Denn in einer Zeit, als die Medizin in ihrer therapeutischen Dimension noch wenig entwickelt war und in der sie häufig kaum mehr Erfolge als laien- oder paramedizinische Massnahmen vorweisen konnte, war es schwer, sich ärztliche Autorität gegenüber dem Kranken aufzubauen und zu erhalten, zumal die Begegnung zwischen Arzt und Patient nicht in der heute

19 Zur Geschichte des Nürnberger Gesundheitswesens vgl. Jegel, August:Werden, Sein und Vergehen des Nürnberger Collegii medici. Bayerische Ärztezeitung 34 (1931) 348-350,360-362, 391-393, 397-398, 409-410; Knefelkamp, Ulrich: Das Heilig-Geist-Spital vom 14.-17. Jahrhundert. Geschichte. Struktur. Alltag. Nürnberg, Verein für Geschichte der Stadt Nürnberg, 1989.

20 Zur Entwicklung der Ärztezahlen in Nürnberg vgl. Jegel (s. Anm. 19), hier S. 397; zur Entwicklung der Bevölkerungsstruktur vgl. Endres, Rudolf: Zur Einwohnerzahl und Bevölkerungsstruktur Nürnbergs im 15./16. Jahrhundert. Mitteilungen des Vereins für Geschichte der Stadt Nürnberg 57 (1970) 242-271; Bog, Ingomar: Wirtschaft und Gesellschaft im Zeitalter des Merkantilismus. In: Pfeiffer, Gerhard (Hrsg.): Nürnberg-Geschichte einer europäischen Stadt. München, Beck, 1971,315-324. 
üblichen dyadischen Vereinzelung im Sprechzimmer des Arztes oder im Untersuchungszimmer eines Krankenhauses, sondern zumeist im Hause des Kranken stattfand und sich dort häufig zu einem sozialen Ereignis gestaltete. Angehörige und gute Freunde, aber auch ärztliche Kollegen oder Vertreter anderer Heilergruppen fanden sich um den Patienten ein und debattierten über das beste medizinische Vorgehen. Der Arzt hatte eine Stimme unter vielen. Vielfach behielt sich der Patient das letzte Wort vor. ${ }^{21}$

Für den Arzt ging es in seiner täglichen Praxis vorrangig darum zu bestehen. Wertet man die Informationen aus, die ein breiteres Studium der Erlanger Briefliteratur liefert ${ }^{22}$, so versuchte er dies auf verschiedenen Wegen. Sein erstes Ziel war es, den Kranken aktiv in die Behandlung einzubinden. So legte er sein Augenmerk darauf, sich verständlich zu machen, indem er seine theoretischen Überlegungen und praktischen Folgerungen gegenüber dem Kranken in umgangssprachlichen Wendungen erläuterte. Weiterhin kontrollierte er den Krankheitsverlauf entweder selbst sehr engmaschig in einem stündlichen, täglichen oder mehrtägigen Rhythmus oder er bat den Patienten, ihn möglichst oft mündlich oder schriftlich über den Stand der Dinge zu informieren. Eine einmal festgelegte Behandlung galt nicht als unumstösslich. Vielmehr versuchte der Arzt, seine Verordnungen, so weit es ging, dem aktuellen Krankheitsbild anzupassen. Dies ermöglichte ihm schliesslich auch, auf die therapeutischen Wünsche des Kranken einzugehen. Dabei zeigte sich der Arzt durchaus als tolerant, auch wenn die Vorstellungen seines Patienten seinen Therapieprinzipien bisweilen grundsätzlich zuwiderliefen. So konnte es passieren, dass der Patient eine Cura sympathetica, eine Wunderkur, vorschlug. Der Arzt liess den Patienten gewähren und kommentierte die Massnahme allenfalls mit den Worten: Ein solches Verfahren wirke immer nur «supra captum», also jenseits aller rationalen Erkenntnis. Letztlich könne nur die Erfahrung den Wert einer solchen Massnahme erweisen. ${ }^{23}$ Somit stand der Arzt des 18. Jahrhunderts in einer städtischen Praxis durchaus nicht auf verlorenem Posten. Bei einigem Geschick und Können vermochte er, sich Achtung und Anerkennung zu verschaffen.

Offen bleibt die Frage, wie der Arzt seine alltägliche Praxis transzendieren und dazu beitragen konnte, die medizinische Theorie voranzutreiben und

21 Vgl. Huerkamp, Claudia: Ärzte und Patienten. Zum strukturellen Wandel der Arzt-PatientBeziehung vom ausgehenden 18. bis zum frühen 20. Jahrhundert. In: Labisch, Alfons und Reinhard Spree (Hrsg.): Medizinische Deutungsmacht im sozialen Wandel des 19. und 20. Jahrhunderts. Bonn, Psychiatrie-Verlag, 1989, 57-73.

22 Vgl. Schnalke,Thomas: Mikrokosmos Trew.Eine Studie über die medizinische Welt des Nürnberger Arztes Christoph Jacob Trew (1695-1769) auf der Grundlage seiner Briefsammlung. Habilitationsschrift, masch.schriftl., Erlangen, 1993.

23 Trew reagiert in diesem Sinne auf eine entsprechende Anfrage eines Patienten; vgl. Scheler P. P. 4, 27. 7. 1747; Trew Chr. J. 669, 1.8.1747. 
zu erläutern. Trew schweigt sich hierüber in seinem Brief an Huth aus. Allerdings zeigt er mit seinem Hinweis auf die verschiedenen Möglichkeiten, mit welchen der städtische Arzt seine erlangten Wissenschaften nutzbringend umsetzen kann, an, dass er in dieser Angelegenheit weitergedacht und differenziertere Ansichten entwickelt hat. Tatsächlich äussert er sich hierzu immer dann konkreter, wenn er ein Angebot ausschlägt, seinen Wirkort Nürnberg zu verlassen, um als Leibarzt an einen Hof oder als Professor an eine Universität zu wechseln. ${ }^{24}$ An dieser Stelle sei eine Passage aus dem Entwurf für ein Schreiben an Johann Lorenz Ludwig Loelius ${ }^{25}$ wiedergegeben, der sich als Ansbachischer Leibarzt 1735 darum bemühte, Trew als einen Kollegen an den Hof zu ziehen: ${ }^{26}$

\section{«[Ich] habe diesem nach die Ehre folgendes gehorsamst zu hinterbringen}

1) Bekenne ich gar gerne daß die Pflicht meinem Vatterlande zu dienen bißhero mich gehindert unterschiedliche vocationes anzunehmen, zumalen da der liebe Gott mich in meinem bißherigen Ampte nachdrücklich also geseegnet hat, daß ich nicht alleine Ihme zum Preiß, meine praxin nun 15 Jahre allhier und vor meinem 3 iährigen Reißen 2 Jahr in Lauff glücklich exerciret, sondern auch, durch unterrichtung der hiesigen studiosorum chirurgiae, bey unterschiedlichen einen solchen grund geleget, daß sie nun mit gutem gewißen ihrem Nächsten dienen können; über dieses auch Liebe und Gnade von meiner hohen Obrigkeit genoßen und so viel, als ich nöthig habe, dabey erworben. Zu diesem allen kommet noch, daß die continuation deß Euer HochEdelgebohren bekanndten und fast in gantz Europa mit allgemeinem beyfall aufgenommenen und vor höchst nützlich erkannten commercii litterarii ohne eitlen Ruhm zu melden, gegenwärtig meinsten [!] theils auf mir lieget; daß ich auch allbereit einen anfang gemacht die beschafenheit deß menschlichen cörpers in einem großen anatomischen werck zu erklären, welche beyde wercke, wann ich eine völlige veränderung meiner gegenwärtigen station vornehmen wollte, nothwendig unterbleiben müsten.»

Dieser Ausschnitt aus dem Briefentwurf Trews weist darauf hin, dass eine Stadt wie Nürnberg im 18. Jahrhundert einem ärztlichen Praktiker genügend Möglichkeiten bot, auch fern der Universität als medizinischer Lehrer, Forscher und Publizist ein quasi akademisches Leben zu führen. Eine wichtige Schlüsselstellung kam hierbei der im Collegium medicum organisierten lokalen Ärzteschaft zu. Sie hatte Rechte und Pflichten in der Verwaltung des territorialen Gesundheitswesens übernommen, wozu etwa die Visitation der Apotheken oder die Ausarbeitung von Empfehlungen zur Sanitätskontrolle gehörten. Darüber hinaus engagierten sich die Nürnberger Ärzte in der Ausbildung von angehenden Wundärzten und Hebammen, sie führten forensische Sektionen und öffentliche anatomische Demonstrationen durch und bemühten sich, durch botanische Studien das pflanzenkundliche Wissen mit

24 Vgl. Trew Chr. J. 237, 30. 8. 1734.

25 Zur Biographie von Johann Lorenz Ludwig Loelius (1687-1756) vgl. v. Vocke, Johann A.: Geburts- und Todten-Almanach Ansbachischer Gelehrter, Schriftsteller und Künstler. 2 Bde. Augsburg, Späth, 1796-1797, 204 und 386.

26 Trew Chr. J. 468, 16. 12. 1735. 
Blick auf die Zusammenstellung wirksamerer Pharmakopoen zu vermehren. $\mathrm{Zu}$ diesen Zwecken unterhielt das Nürnberger Collegium medicum ein anatomisches Theater sowie einen botanischen Garten, deren Beaufsichtigung zumeist für einige Jahre in den Händen eines jüngeren, ambitionierten Kollegen lag. Christoph Jacob Trew übernahm diese Aufgabe 1721 und hielt in den 1720er Jahren an diesen Einrichtungen Lehrsektionen, chirurgisch-theoretische Übungen und botanische Exkursionen ab. ${ }^{27}$

Aus den Reihen seiner Hörer erwuchs Trew im Laufe der Zeit ein Kreis von Mitarbeitern, auf die er später für unterschiedliche Zwecke zurückgreifen konnte. So zog er sich Chirurgen seines Vertrauens heran, die nicht nur die äusserliche Therapie selbständig, mit grosser Könnerschaft und verantwortungsbewusst betrieben, sondern die auch loyal für die eine oder andere von Trew durchgeführte Behandlung mit dem Arzt kooperierten und sich bisweilen sogar als dessen Assistenten einsetzen liessen. ${ }^{28}$

Unter den Kunstschaffenden, die den medizinischen Übungen Trews beiwohnten, fanden sich Zeichner, Maler, Stecher und Illuminatoren, die nach neuen Einsatzbereichen für ihre Talente suchten und ihre illustratorischen Dienste auch den Wissenschaften anboten. Mit ihrer Hilfe versuchte Trew später, aufwendige Abbildungswerke zu realisieren. Darüber hinaus regte er seine Künstlerschüler zu eigenständigem naturkundlichen Beobachten, Studieren und Zeichnen an und ermunterte sie, selbst Editionsvorhaben zu entwickeln, um auf diese Weise einen Nutzen für die Allgemeinheit zu stiften. ${ }^{29}$

Bei der Arbeit auf dem Theatrum anatomicum und im Hortus medicus in Nürnberg fielen für den praktischen Arzt eine Reihe von interessanten Objekten an: Präparate, Instrumente, Pflanzen und Pflanzensamen. Es lag nahe, diese Realien aufzuheben und zu sammeln. Dies tat auch Trew. Zunächst sammelte er dingliche Zeugnisse, später kamen Abbildungen hinzu, vor allem von Pflanzen, die ihm eine Reihe von Künstlern auf seinen Wunsch hin ausführten, und schliesslich dehnte Trew sein Sammelinteresse auf Bücher, Briefe und ganze Korrespondenzen von sich selbst, mehr oder weniger bekannten Zeitgenossen und bedeutsamen Gelehrten früherer Epochen aus. ${ }^{30}$

27 Vgl. Schmidt-Herrling (Anm. 1) 640:Trew Chr. J. (Amtliche Berichte und Eingaben an Nürnbergische Behörden), Beilagen b-f, 17.3.1723; 5. 7.1723;24.1.1724; 20. 9.1724, 25. 7.1730.

28 Vgl. die Zusammenarbeit zwischen Trew und dem Nürnberger Chirurgen Johann Eberhard Eyrich; Eyrich J. E. 1, 7. 6. 1747; Eyrich J. E. 2, 17. 7. 1747; Trew 463, o.D.

29 Vgl. Nissen, Claus: Die botanische Buchillustration. Ihre Geschichte und Bibliographie. 1. Bd. Stuttgart, Hiersemann, 1951, 167-168; Mann, Gunter: Medizinisch-naturwissenschaftliche Buchillustration im 18. Jahrhundert in Deutschland. Sitzungsberichte der Gesellschaft zur Beförderung der gesamten Naturwissenschaften zu Marburg 84 (1964) 3-48; Ludwig, Heidrun: Nürnberger naturgeschichtliche Malerei im 17. und 18. Jahrhundert. Berlin, Diss. phil., 1993.

30 Vgl. Schmidt-Herrling, Eleonore: Die Bibliothek des Arztes Christoph Jacob Trew. In: Werner, Gunda und Eleonore Schmidt-Herrling: Die Bibliotheken der Universität Altdorf. Leip- 
Als Sammler war Trew ein Kind seiner Zeit. Er reihte sich unter die Gelehrten und akademisch gebildeten Ärzte, die sich im 18. Jahrhundert aus einem dezidiert naturkundlichen Interesse Privatsammlungen aufbauten. Sie sammelten nicht zur privaten Erbauung, vielmehr bündelten sie das Wissen ihrer Zeit und ihres Gebietes in ihren eigenen vier Wänden, um damit zu arbeiten, zu forschen und daraus auch zu publizieren. Indem sie so die Hermetik ihrer Sammlungen durchbrachen und ihre Schätze wieder Allgemeingut werden liessen, fanden sie zu einem eigenen, ihnen gemässen wissenschaftlichen Ansatz.

Dass die wissenschaftliche Gemeinschaft die so geartete Forschungsbeteiligung des praktischen Arztes durchaus zu schätzen wusste, hatte seine Gründe in der Medizin der Zeit. In der ersten Hälfte des 18. Jahrhunderts ging es für diese noch sehr darum, Befunde, Beobachtungen und Ergebnisse zu sammeln, zu sortieren und zu systematisieren. Dabei lässt sich das Streben um neue Klassifikationen am besten auf dem Gebiet der Botanik verfolgen, die in jener Zeit noch ein integraler Bestandteil der Medizin war. Zwar setzte sich bekanntermassen das binäre Klassifikationsmodell Carl von Linnés in der botanischen Nomenklatur durch, doch neben Linné rang beinahe die gesamte medizinische Forschergeneration der Zeit um neue, bessere Systematiken für das Reich der Pflanzen. Hieran beteiligten sich Gelehrte wie der Göttinger Universitätsprofessor Albrecht von Haller, aber auch praktische Ärzte im Range eines Trew. ${ }^{31}$

Trew schuf sich mit seinen Sammlungsgruppen seine Arbeitsgrundlage, auf der er sich als praktischer Arzt an der wissenschaftlichen Diskussion seiner Zeit beteiligen konnte. Dabei mag es so scheinen, als ob er im Sinne Kuhns kein originärer Forscher war, der nach paradigmatisch neuen Ufern strebte. Dieser Auffassung dürfte sich Trew mit einiger Berechtigung nicht angeschlossen haben. Er sah sein Tun durchaus als ein Hinzufügen neuer Erkenntnisbausteine auf die Mauern einer zu errichtenden, verbesserten medizinischen Wissenschaft. In diesem Bewusstsein wurde er gestärkt durch einen Trend in der Auffassung von Wissenschaft in der Frühzeit der medizinischen Aufklärung. Danach bewegte sich der medizinische Fortschritt vor allem in den Bahnen einer evolutionären Entwicklung, die stark in der medizinischen Praxis gründete. ${ }^{32}$ Die Einzelbeobachtung zählte, die Studie einer Pflanze etwa, aber auch, um wieder in die klinische Medizin zurückzukehren, die Beobachtung am Krankenbett. Wichtig war die davon niederge-

zig, Harrassowitz, 1937, S. 88-138; dies. (s. Anm. 1), Einleitung, S. XI-XLIII; Pirson (s. Anm. 8) 467-477.

31 Vgl. in diesem Zusammenhang Schnalke (s. Anm. 22) 49-85.

32 Vgl. Geyer-Kordesch 1990 (s. Anm. 2), Müller (s. Anm. 2).

4 Gesnerus 
legte, minutiös dokumentierte Krankengeschichte. Kasuistiken erschienen in grosser Zahl auf dem Fachbuchmarkt. Führende Persönlichkeiten der medizinischen Gelehrtenwelt, von Friedrich Hoffmann und Georg Ernst Stahl bis zu Lorenz Heister, beteiligten sich mit zum Teil mehrbändigen Werken an dieser Literaturgattung. ${ }^{33}$ Sie sahen den Wert dieser Schriften nur zum Teil in ihrer didaktischen Funktion für den angehenden Arzt. Vor allem vertraten sie die Ansicht, dass sich aus dem Studium der Praxis im Einzelfall wissenschaftliche Erkenntnisse im Allgemeinen gewinnen lassen.

In diesem Klima, das die Wahrnehmung des Einzelfalls in der Praxis so hoch ansetzte, fühlte sich insbesondere der niedergelassene Arzt dazu ermuntert, seine Erfahrungen in die wissenschaftlichen Debatten der Zeit einfliessen zu lassen. Die Frage stellt sich allerdings, in welcher Form er sich am gelehrten Fachaustausch beteiligen konnte. Er war ja nicht an einer Universität beschäftigt, wo er sich ganz dem wissenschaftlichen Arbeiten und Publizieren widmen und sich der notwendigen Veröffentlichungskanäle bedienen konnte. Eine grössere Stadt wie Nürnberg bot auch in dieser Hinsicht durch die Einrichtung eines Collegium medicum Hilfestellung. Eine verfasste Ärzteschaft erleichterte den wissenschaftlichen Dialog nach innen wie nach aussen. Einerseits konnte ein praktischer Arzt seine anatomischen, botanischen und klinischen Observationes intern, im Kreis seiner Kollegen, diskutieren. Andererseits vermittelten ihm die Kontakte, die ihm seine bereits etablierten Kollegen verschafften, die Möglichkeit, sich ein Netz von persönlichen Beziehungen aufzubauen, über welche er sein Sammeln und Forschen organisieren und seine Wahrnehmungen in überregionalen oder gar internationalen Kreisen diskutieren konnte. Hierfür war es bedeutsam, dass die Gelehrtenwelt des 18. Jahrhunderts als grenzenlose Korrespondentenrepublik vernetzt und die Einrichtung und Führung einer Fachkorrespondenz an keine gelehrte Institution gebunden war. ${ }^{34}$

Unter seinen Nürnberger Kollegen fand Trew zudem die notwendigen Mitstreiter, um sich ein eigenes Forum zu schaffen, auf dem er seine Beob-

33 Vgl. Hoffmann, Friedrich: Medicina consultatoria: worinnen unterschiedliche und einige schwehre Casus ausgearbeitete Consilia, auch Responsa Facultatis Medicae enthalten, und in fünff Decurien eingetheilet. 1.-12. Theil. Halle, Renger, 1721-1739; Stahl, Georg Ernst: Collegium casuale magnum: oder sechs und siebentzig practische Casus, welche er von Anno 1705 bis 1707 als Professor Ordinarius auf der Universität Halle einem gewissen numero studiosorum [...] lateinisch in die Feder dictirt, nunmehro ins Deutsche übersetzt von Johann Storchen. Leipzig, Eyssel, 1733; Heister, Lorenz: Medicinische Chirurgische und Anatomische Wahrnehmungen. Rostock, Koppe, 1753.

34 Vgl. Wojowitz, J.: Korrespondentenzirkel als Kommunikationsgruppen im Zeitalter der Aufklärung. Vorschläge - Postulate - Forschungsmöglichkeiten. In: Dutu, A., E. Hösch und N. Oellers (Hrsg.): Brief und Briefwechsel in Mittel- und Osteuropa im 18. und 19. Jahrhundert. Essen, Reimar Hobbing, 1989, 271-282. 
achtungen veröffentlichen konnte. 1730 gehörte er zu den Gründungsmitgliedern einer fünfköpfigen Sozietät ${ }^{35}$, die zwischen 1731 und 1745 eine wöchentlich erscheinende medizinische Zeitschrift, das «Commercium litterarium ad rei medicae et scientiae naturalis incrementum», herausgab. Trotz grosser Schwierigkeiten fand das Periodikum in ganz Europa Verbreitung und genoss zeitweise grosses Ansehen. ${ }^{36}$

Mit dem Commercium litterarium schuf sich Trew nicht nur selbst eine Möglichkeit für Veröffentlichungen, sondern er gewann als Kontribuenten neben angesehenen Fachgelehrten auch ärztliche Praktiker. Damit schlüpfte er, zumal ab 1734 die Hauptlast der Herausgebergeschäfte auf ihm ruhte, in die Rolle eines zentralen Mediators im medizinisch-wissenschaftlichen Kommunikationsfluss seiner Zeit.

Eine Stadt wie Nürnberg bot dem wissenschaftlich ambitionierten Arzt noch weiterreichende Publikationsmöglichkeiten. In seinem Veröffentlichungsdrang war dieser jedoch nicht nur auf eingeführte Verlage angewiesen. Vielmehr fanden sich wissenschaftliche Illustratoren dazu bereit, das finanzielle Risiko zu übernehmen und im Eigenverlag grössere Tafelwerke zu publizieren, für welche sie die Abbildungen gestaltet und gelehrte Autoren den Text verfasst hatten. ${ }^{37}$ Trew wusste dieses Engagement zu fördern und davon zu profitieren. Zu Beginn der 1730er Jahre entwickelte er sein Vorhaben, ein sechs- bis achtbändiges illustriertes Opus anatomicum herauszubringen, ein Projekt, das er nur als ein Fragment des ersten Teils, die Osteologie des Schädels, verwirklichen konnte. ${ }^{38} \mathrm{Ab}$ den späten $1740 \mathrm{er}$ Jahren richtete er sein ganzes Augenmerk auf die Herausgabe prachtvoll ausgestatteter, grossformatiger, illustrierter Pflanzenwerke.

In den von ihm edierten botanischen Foliobänden gelang es Trew, sein wissenschaftliches Streben zu bündeln, seinen Forschungsansatz umzusetzen und den ihm eigenen publizistischen Präsentationsstil zur Perfektion zu entwickeln. Seine Sammlungen lieferten ihm dafür die Grundlagen. Er besass inzwischen zahllose Pflanzenzeichnungen, -aquarelle, -stiche, prachtvolle Atlanten ausländischer Provenienz sowie eine reichbestückte Bibliothek, die ihm das pflanzenkundliche Wissen seiner Zeit in aller Breite erschloss. Er sichtete diese Bestände, wählte publikationswürdige Stücke für einzelne Buchprojekte aus und bereitete sie teilweise selbst wissenschaftlich auf. Die

35 Hierzu zählten neben Trew die drei Nürnberger Ärzte Johann Christoph Götz (1688-1733), Johann Christoph Homann (1703-1730) und Christoph Wilhelm Preissler (1702-1734), sowie der Altdorfer Professor Johann Heinrich Schulze.

36 Zum Schicksal des Commercium litterarium vgl. Pirson (s. Anm. 8) 477-490.

$37 \mathrm{Vgl}$. Anm. 29.

38 Trew, Christoph Jacob: Osteologie oder eigentliche Fürstellung und Beschreibung aller Beine eines erwachsenen Menschlichen Cörpers. Nürnberg, Lichtensteger und Eisenberger, 1740. 
von ihm in die Naturkunde eingeführten Künstler dienten ihm als Vermittler seiner gleichermassen wissenschaftlichen wie illustratorisch-ästhetischen Ansprüche. Unter seiner Anleitung führten sie neue Zeichnungen oder Aquarelle aus oder übersetzten die Vorlagen in druckfertige Kupferstichvorlagen. Trew öffnete seine Sammlung aber auch angesehenen botanischen Gelehrten, die grössere Corpora bearbeiteten und veröffentlichten. Unter diesen von ihm verwirklichten oder angeregten Werken finden sich Druckerzeugnisse, die heute zu den Meisterleistungen der illustrierten botanischen Buchproduktion des 18. Jahrhunderts im deutschen Sprachraum gerechnet werden ${ }^{39}$ Erwähnt seien hier lediglich Trews «Plantae selectae», seine Überarbeitung des «Herbarium Blackwellianum», der «Hortus nitidissimus» sowie seine «Plantae rariores».40

\section{Fazit}

Das Beispiel Trews richtet den Blick auf einige Merkmale des beruflichen Wirkens eines Arztes, der sich im 18. Jahrhundert in einer grösseren Stadt niederliess. In einer oft schwierigen Praxissituation konnte sich dieser gegenüber seinen Patienten Achtung und medizinische Autorität verschaffen, wenn er vor allem drei Ziele verfolgte:

- Er musste versuchen, die kritische Phase seines beruflichen Einstiegs durch ein auf die jeweilige Situation angepasstes Verhalten, das in einem abgewogenen Verhältnis zwischen medizinischem Handeln und ärztlicher Zurückhaltung bestand, zu meistern.

- Er musste sich darauf einlassen, seine ihm aus der täglichen Arbeit zuwachsende Erfahrung stets produktiv vor dem Hintergrund seines theoretischen Wissens zu messen, zu wägen und diese durch einen solch gearteten inneren Schulungsprozess in immer gesichertere Verhaltens- und Handlungsstrategien umzusetzen.

39 Vgl. Wegner, Richard N.: Christoph Jacob Trew (1695-1769). Ein Führer zur Blütezeit naturwissenschaftlicher Abbildungswerke in Nürnberg im 18. Jahrhundert. Mitteilungen zur Geschichte der Medizin, der Naturwissenschaften und der Technik 39 (1940) 218-228; Nissen (s. Anm. 29); Mann (s. Anm. 29).

40 Trew, Christoph Jacob: Plantae selectae quarum imagines ad exemplaria naturalia Londini in hortis curiosorum nutrita manu artificiosa doctaque pinxit Georgius Dionysius Ehret. Augsburg, 1750-1773; ders.: Herbarium Blackwellianum emendatum et auctum id est Elisabethae Blackwell collectio stirpium. 6 Bde. Nürnberg, Eisenberger, 1750-1773; ders.: Hortus nitidissimus, omnem per annum suberbiens floribus, sive amoenissimarum florum imagines. 3 Bde. Nürnberg, Seligmann und Wirsing, 1750-1786; ders.: Plantae rariores. Decas I-III. Nürnberg, Keller und Wirsing, 1763-1784. 
- Es musste ihm daran gelegen sein, durch eine ausführliche umgangssprachliche Erläuterung seiner medizinischen Überlegungen und Verordnungen, durch eine engmaschige Kontrolle des Befundes und eine dementsprechend modifizierte und somit immer wieder aktualisierte Therapie sowie durch ein weitreichendes Eingehen auf die Behandlungswünsche des Kranken eine grösstmögliche Patientenbindung herzustellen.

Neben seiner städtischen Praxis war es einem Arzt im 18. Jahrhundert möglich, abseits der Universitäten ein quasi akademisches Leben zu führen und sich am wissenschaftlichen Austausch seiner Zeit zu beteiligen. Hierfür erwiesen sich vor allem drei Voraussetzungen als förderlich:

- Die Stadt musste die notwendigen wissenschaftlichen Infrastrukturen bereitstellen. Günstigerweise verfügte sie über ein Collegium medicum, das eigene Forschungseinrichtungen unterhielt und die Fachdiskussion nach innen sowie nach aussen, etwa durch die Gründung von Fachzeitschriften, förderte. Zudem war es von Vorteil, wenn in der Stadt eingeführte Fachverlage ansässig waren sowie eine Reihe von Künstlern ihr Auskommen suchten, die bereit waren, ihre Talente in die Dienste der naturkundlichen Illustration zu stellen und manche Werke im Eigenverlag zu publizieren.

- Der wissenschaftlich ambitionierte Praktiker musste danach trachten,Anschluss an das grenzenlose Korrespondentennetz der Gelehrtenrepublik seiner Zeit zu finden. Auch hierbei konnte eine eingeführte lokale Kollegenschaft wichtige Vermittlerdienste leisten.

- Für den Arzt erwies sich der Besitz einer naturkundlichen Sammlung als wesentliches Mittel seiner Forschung. In ihr konnte er das zu seiner Zeit verfügbare Wissen auf seinen Forschungsgebieten zusammentragen, sichten und es mit seinen aus der klinischen Erfahrung gespeisten Beobachtungen und Überlegungen vergleichen, um daraus seine Einsichten zu publizieren.

Unter diesen Bedingungen war der Arzt in einer grösseren Stadt in der Lage, das in der Praxis verankerte und am theoretischen Wissen geläuterte Erkennen zu einem wichtigen heuristischen Movens in der Medizin des 18. Jahrhunderts zu kultivieren. 